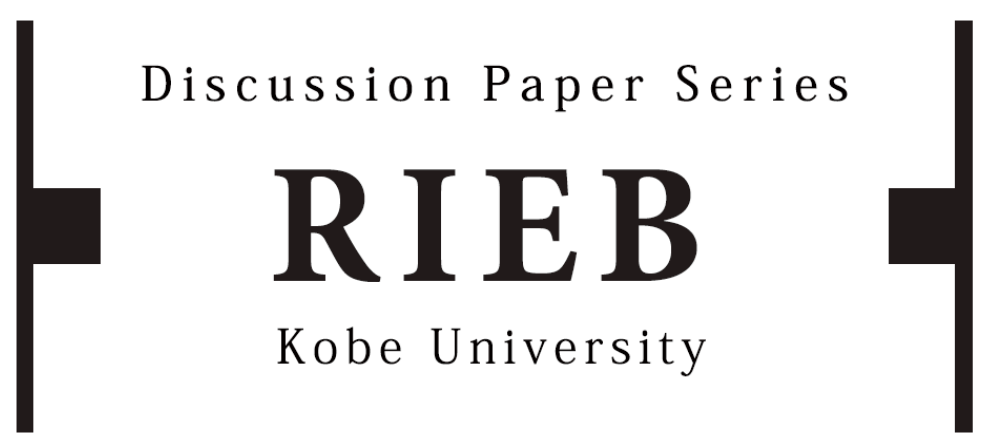

DP2011-19

Discrete Choice and Complex Dynamics in Deterministic Optimization Problems

Takashi KAMIHIGASHI

Revised July 13, 2011

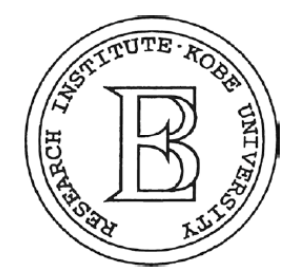

Research Institute for Economics and Business Administration Kobe University 


\title{
Discrete Choice and Complex Dynamics in Deterministic Optimization Problems*
}

\author{
Takashi Kamihigashi ${ }^{\dagger}$ \\ July 13, 2011
}

\begin{abstract}
This papers shows that complex dynamics arise naturally in deterministic discrete choice problems. In particular, we show that if the objective function of a maximization problem can be written as a function of a sequence of discrete variables, and if the (maximized) value function is strictly increasing in an exogenous variable, then for almost all values of the exogenous variable, any optimal path exhibits aperiodic dynamics. This result is applied to a maximization problem with indivisible durable goods as well as to a Ramsey model with an indivisible consumption good. In each model, it is shown that optimal dynamics are almost always complex. These results are illustrated with various numerical examples.
\end{abstract}

Keywords: Discrete choice, complex dynamics, chaos, indivisible goods.

*This paper has benefited from comments by an anonymous referee. Financial support from the Japan Economic Research Foundation and the Japan Society for the Promotion of Science (KAKENHI No. 21243027) is gratefully acknowledged.

${ }^{\dagger}$ RIEB, Kobe University, Rokkodai, Nada, Kobe 657-8501 JAPAN. Email: tkamihig@rieb.kobe-u.ac.jp. Tel/Fax: +81-78-803-7015. 


\section{Introduction}

Discrete choice problems abound in economic decision making. Most manufactured products, real estates, and works of art are indivisible. Choices regarding education, occupation, marriage, etc. are discrete in nature. It is not surprising that over the past decades, dynamic discrete choice models have gained considerable popularity in empirical studies (e.g, Keane and Wolpin, 2009; Aguirregabiria and Mira, 2010).

In sharp contrast to the popularity of these models, there have been very few developments regarding deterministic, dynamic discrete choice problems since Kamihigashi (2000a, 2000b). ${ }^{1}$ The purpose of this paper is to reinforce the point made in our earlier work that complex dynamics arise rather naturally in deterministic discrete choice models. ${ }^{2}$ In particular, we show that if the objective function of a maximization problem can be expressed as a function of a sequence of endogenous discrete variables, and if the value function (or maximized value of the objective function) is strictly increasing in an exogenous variable, then for almost all values of the exogenous variable, any optimal path exhibits aperiodic dynamics.

This result generalizes the similar result shown in Kamihigashi (2000a) for a life-cycle model with an indivisible consumption good. Another similar result was shown in Kamihigashi (2000b) for an optimization problem with indivisible labor. While these previous results were shown for specific models, the result shown in this paper is extremely general and can be applied to a wide range of economic problems. For example, we apply this result to an optimization problem with indivisible durable goods as well as to a Ramsey model with an indivisible consumption good, and show that in these models, optimal paths almost always exhibit complex dynamics. These results are illustrated with various numerical examples.

This paper is related to two branches of the literature on the optimal dynamics of deterministic models. First, since discrete choice problems can be regarded as models with nonconvexities, this paper adds to the literature on the discrete-time dynamics of optimal growth with nonconvexities, which was initiated by the seminal paper of Dechert and Nishimura (1983). ${ }^{3}$ Sec-

\footnotetext{
${ }^{1}$ See Aoki (1998), Verbrugge (2003), and Bischi et al. (2006) for examples of theoretical stochastic models involving discrete choices.

${ }^{2}$ In this paper, the term "complex" means "aperiodic and bounded."

${ }^{3}$ Their analysis was recently extended by Kamihigashi and Roy $(2006,2007)$ to models with nonsmooth technologies.
} 
ond, this paper is also related to the literature on the possibility of chaos in optimal growth models with low discounting (e.g., Nishimura and Yano, 1995; Nishimura, Yano, and Sorger, 1994), since our result shows that the optimal dynamics of a discrete choice model can be complex for any discount factor. ${ }^{4}$

The rest of the paper is organized as follows. Section 2 establishes our general result. Section 3 illustrates this result with a model with an indivisible consumption good as well as one with indivisible durable goods. Section 4 studies a Ramsey model with an indivisible consumption good.

\section{The General Result}

Let $n \in \mathbb{N}$. Let $\lambda(A)$ denote the Lebesgue measure of the (Lebesgue) measurable set $A \subset \mathbb{R}$, and $\lambda^{n}(B)$ the Lebesgue measure of the measurable set $B \subset \mathbb{R}^{n}$. For $j=1, \ldots, n$, let $I_{j}$ be an interval in $\mathbb{R}$ with nonempty interior. Each $I_{j}$ need not be closed and need not be bounded. Let $X=\prod_{j=1}^{n} I_{j}$. Let $K$ be a nowhere dense (thus countable) subset of $\mathbb{R}^{m}$ with $m \in \mathbb{N}$. Define $K^{\infty}$ to be the set of sequences in $K: K^{\infty}=\left\{\left\{c_{t}\right\}_{t=0}^{\infty}: \forall t \in \mathbb{Z}_{+}, c_{t} \in K\right\}$. Let $C$ be a correspondence from $X$ to $K^{\infty}$; i.e., $\forall x \in X, C(x) \subset K^{\infty}$. Define $D=\cup_{x \in X} C(x)$. Let $w: D \rightarrow \mathbb{R}$. We assume the following.

Assumption 2.1. For each $x \in X, \max _{c \in C(x)} w(c)$ exists in $\mathbb{R}$.

For $x \in X$, define

$$
\begin{gathered}
v(x)=\max _{c \in C(x)} w(c), \\
C^{*}(x)=\underset{c \in C(x)}{\operatorname{argmax}} w(c) .
\end{gathered}
$$

We use the following definitions: a function $g: \mathbb{R}^{n} \rightarrow \mathbb{R}$ is strictly increasing if $g(x)<g(y)$ whenever $x<y ;^{5}$ a sequence $\left\{z_{t}\right\}_{t=0}^{\infty}$ (in $K$ or $\mathbb{R}^{n}$ ) is periodic if there exists $i \in \mathbb{N}$ such that $\forall t \in \mathbb{Z}_{+}, z_{t+i}=z_{t}$; a sequence $\left\{y_{t}\right\}$ is eventually periodic if there exists a periodic sequence $\left\{z_{t}\right\}$ such that $\exists T \in \mathbb{Z}_{+}, \forall t \geq T, y_{t}=z_{t}$; a sequence $\left\{y_{t}\right\}$ is asymptotically periodic if there exists a periodic sequence $\left\{z_{t}\right\}$ such that $\left\|y_{t}-z_{t}\right\| \rightarrow 0$ as $t \uparrow \infty$, where $\|\cdot\|$

\footnotetext{
${ }^{4}$ See Kamihigashi (2000b) for discussion on ergodic chaos in a discrete choice model.

${ }^{5}$ Here the inequality $x<y$ means that $x^{i} \leq y^{i}$ for all $i=1, \ldots, n$, and there is at least one $i$ with $x^{i}<y^{i}$, where $x=\left(x^{1}, \ldots, x^{n}\right)$, etc.
} 
is any equivalent norm; and a sequence $\left\{y_{t}\right\}$ is asymptotically aperiodic if it is not asymptotically periodic. Since $K$ is nowhere dense, a sequence in $K$ is asymptotically aperiodic if and only if it is not eventually periodic.

We are ready to state the main result of this paper:

Theorem 2.1. Suppose that $v: X \rightarrow \mathbb{R}$ is strictly increasing. ${ }^{6}$ Then for almost all $x \in X$ (with respect to Lebesgue measure), any sequence $\left\{c_{t}\right\} \in$ $C^{*}(x)$ is asymptotically aperiodic. In particular, there exists a measurable set $Z \subset X$ such that (a) $\lambda^{n}(Z)=0$, and (b) for each $x \in X \backslash Z$, any sequence $\left\{c_{t}\right\} \in C^{*}(x)$ is asymptotically aperiodic.

Proof. See Appendix A.

This result considerably generalizes Kamihigashi (2000a, Theorem 2). The proof of Theorem 2.1 shows that the set of eventually periodic sequences in $K$ is countable. The assumption that $v$ is strictly increasing ensures that for each $\left\{c_{t}\right\} \in K^{\infty}$, the set of $x \in X$ with $v(x)=w\left(\left\{c_{t}\right\}\right)$ has measure zero. Since the set of eventually periodic sequences in $K$ is countable, it follows that the set of $x \in X$ with $v(x)=w\left(\left\{c_{t}\right\}\right)$ for some eventually periodic sequence $\left\{c_{t}\right\} \in K^{\infty}$ has measure zero.

The only role of the value function in the proof is to ensure that for each $\left\{c_{t}\right\} \in K^{\infty}$, the set of $x \in X$ with $v(x)=w\left(\left\{c_{t}\right\}\right)$ has measure zero. Thus the conclusions of Theorem 2.1 hold for an arbitrary correspondence $C^{*}$ from $X$ to $K^{\infty}$ such that for each $\left\{c_{t}\right\} \in K^{\infty}$, the set of $x \in X$ with $\left\{c_{t}\right\} \in C^{*}(x)$ has measure zero. If $C^{*}$ is given by $(2)$, this can be ensured by assuming that each level set of $v$ has measure zero:

Corollary 2.1. Suppose that $v: X \rightarrow \mathbb{R}$ is measurable. Suppose further that

$$
\forall a \in \mathbb{R}, \quad \lambda^{n}(\{x \in X: v(x)=a\})=0 .
$$

Then the conclusions of Theorem 2.1 hold.

Proof. See Appendix A.

\footnotetext{
${ }^{6}$ Given our assumption on $X$, the value function $v$ is also measurable by Chabrillac and Crouzeix (1987, Theorem 4). The proof of Lemma A.1 shows that we need only assume that $v$ is nondecreasing and that there is at least one $i$ such that $v\left(x_{1}, \ldots, x_{n}\right)$ is strictly increasing in $x_{i}$.
} 


\section{$3 \quad$ Examples}

\subsection{Rational vs. Irrational Numbers}

To better understand Theorem 2.1, consider the following rather trivial maximization problem:

$$
\begin{aligned}
\max _{\left\{c_{t}\right\}_{t=0}^{\infty}} & \sum_{t=0}^{\infty} 10^{-t} c_{t} \\
\text { s.t. } & \sum_{t=0}^{\infty} 10^{-t} c_{t} \leq x, \\
\forall t \in \mathbb{Z}_{+}, & c_{t} \in\{0,1,2, \ldots, 9\} .
\end{aligned}
$$

We assume that $x \in X \equiv[0,10]$. It is immediate from (5) that the value function $v(x)$ for this problem satisfies $v(x) \leq x$. Since $x$ has a decimal representation $x=c_{0} \cdot c_{1} c_{2} c_{3} \ldots$ satisfying (5) with equality, we also have $v(x) \geq x$. Hence $v(x)=x$, and $C^{*}(x)$ can be identified with the decimal representations of $x$. Clearly $v$ is strictly increasing, so that Theorem 2.1 applies.

Recall that a real number has an eventually periodic decimal representation if and only if it is rational. Let $Z$ be the set of rational numbers in $X$. Since $Z$ is countable, we have $\lambda(Z)=0$. If $x \in X \backslash Z$, then $x$ is irrational, and the decimal representation of $x$ is asymptotically aperiodic. It follows that $Z$ has properties (a) and (b) in Theorem 2.1.

\subsection{Indivisible Consumption Goods}

Consider the following maximization problem:

$$
\begin{array}{cl}
\max _{\left\{c_{t}, s_{t}, x_{t+1}\right\}_{t=0}^{\infty}} & \sum_{t=0}^{\infty} \beta^{t} u\left(c_{t}\right) \\
\text { s.t. } \forall t \in \mathbb{Z}_{+}, & c_{t}+s_{t}=x_{t}, \\
& x_{t+1}=R s_{t}+y, \\
& x_{t+1} \geq 0, \\
& c_{t} \in\{0, \delta, 2 \delta, 3 \delta, \ldots\}, \\
& x_{0}=x \geq 0 \text { given, }
\end{array}
$$


where $u: \mathbb{R}_{+} \rightarrow \mathbb{R}$ is the utility function, which is assumed to be strictly increasing; $\beta \in(0,1)$ is the discount factor; $c_{t}$ is the consumption of the indivisible good in period $t ; x_{t}$ is wealth at the beginning of period $t ; s_{t}$ is saving in period $t$; and $R>1$ and $y \geq 0$ are the gross interest rate and income in each period, respectively. Constraint (12) means that the consumption good can be purchased only in multiples of $\delta$. As $\delta \downarrow 0$, this problem approaches the standard problem with $c_{t} \geq 0$ instead of (11). ${ }^{7}$

In this model the consumption good is indivisible, while wealth is perfectly divisible. It is necessary for wealth to be a continuous variable as long as the gross interest rate $R$ is an arbitrary real number strictly greater than one. In most cases it seems reasonable to assume that wealth is more divisible than goods, and our setup represents the extreme case in which wealth is perfectly divisible.

We say that a three dimensional sequence $\left\{c_{t}, s_{t}, x_{t+1}\right\}$ is a feasible path from $x$ if it satisfies (8)-(12), and an optimal path from $x$ if it solves the maximization problem (7)-(12); a sequence $\left\{c_{t}\right\}$ is a feasible consumption path from $x$ if there exist sequences $\left\{s_{t}\right\}$ and $\left\{x_{t+1}\right\}$ such that $\left\{c_{t}, s_{t}, x_{t+1}\right\}$ is feasible from $x$. Optimal consumption paths, feasible wealth paths, and optimal wealth paths are defined similarly. We say that $x \geq 0$ is a steady state if the wealth path $\left\{x_{t+1}\right\}$ with $x_{t}=x$ for all $t \in \mathbb{N}$ is optimal from $x$.

Under regularity conditions, an optimal path from $x$ exists and the value function $v(x)$ is finite for all $x \geq 0$. We assume these properties in what follows.

To see that $v$ is strictly increasing, let $0 \leq x<x^{\prime}$, and let $\left\{c_{t}, s_{t}, x_{t+1}\right\}$ be an optimal path from $x$. Note that $\left\{c_{t}\right\}$ is feasible also from $x^{\prime}$. Thus $v(x) \leq v\left(x^{\prime}\right)$. Let $\left\{c_{t}^{\prime}, s_{t}^{\prime}, x_{t+1}^{\prime}\right\}$ be the feasible path from $x^{\prime}$ with $\left\{c_{t}^{\prime}\right\}=\left\{c_{t}\right\}$. It follows from (8) and (9) that

$$
\forall t \in \mathbb{Z}_{+}, \quad x_{t}^{\prime}-x_{t}=R^{t}\left(x-x^{\prime}\right) .
$$

Since $R>1$, we have $x_{t}^{\prime}-x_{t}>\delta$ for sufficiently large $t$, which implies that it is feasible to increase $c_{s}^{\prime}$ for some large $s$ without decreasing any $c_{t}^{\prime}$ with $t \neq s$. It follows that $v$ is strictly increasing. Now by Theorem 2.1, for almost all $x \geq 0$, any optimal consumption path from $x$ is asymptotically aperiodic. This implies that the corresponding wealth path is also asymptotically aperiodic. ${ }^{8}$

\footnotetext{
${ }^{7}$ Essentially the same model is studied in Kamihigashi (2000a). Here we offer additional insight as well as outlining some of the basic arguments in Kamihigashi (2000a) to facilitate subsequent discussion.

${ }^{8}$ Note that given any feasible path $\left\{c_{t}, s_{t}, x_{t+1}\right\}$, if $\left\{x_{t}\right\}$ is asymptotically periodic,
} 
The above result is shown in Kamihigashi (2000a), where it is also shown that for $\beta$ sufficiently small, the (optimal) policy function for wealth takes the form of a random number generator. The bottom plot in Figure 1(a) illustrates a policy function for wealth that takes the form of a linear congruential generator. ${ }^{9}$ Recall however that Theorem 2.1 does not require $\beta$ to be small. The bottom plot in Figure 1(b) shows a policy function for wealth with $\beta=0.7$. In this case, although there is an overall tendency for wealth to decline toward zero, it keeps fluctuating near zero, which is not a steady state. Figure 2 illustrates optimal wealth paths in the two cases in Figure 1.

The assumption that $R>1$ plays two important roles here. First, it is crucial to showing that $v$ is strictly increasing; recall (13). Second, it implies that any steady state is locally unstable. This is particularly clear in Figure 1(a). Indeed, whenever the policy function for wealth crosses the 45 degree line, it does so from below. This is because we have $x_{t+1}=R\left(x_{t}+y-c_{t}\right)$ by (8) and (9), and the policy function for wealth is continuous only where the policy function for consumption is constant, which implies that the slope of the former is $R>1$ wherever it is continuous.

If $R<1$, then $v$ is never strictly increasing. This is illustrated in Figure 3(a), which shows a piecewise constant value function. If $R<1$, wealth shrinks to zero even if nothing is consumed. Thus consumption, being discrete, can take place only finitely many times. Hence any compact interval can be divided into finitely many subintervals according to optimal consumption paths. It is also interesting to observe that consumption is clearly not a monotone function of wealth in Figure 3(a).

As discussed above, Theorem 2.1 applies as long as $R>1$. This however does not mean that optimal dynamics are almost always complex. For example, if $y=0$, the maximization problem here is an AK model, and endogenous growth is possible depending on the parameter values. Such a case is illustrated in Figure 3(b), ${ }^{10}$ where both consumption and wealth grow un-

then $\left\{c_{t}\right\}$ must be asymptotically periodic by (8) and (9). Hence if $\left\{c_{t}\right\}$ is asymptotically aperiodic, then $\left\{x_{t}\right\}$ must be asymptotically aperiodic.

${ }^{9}$ See Kamihigashi (2000a) for discussion on random number generators. Each numerical example in this paper (except for Figure 3(b)) is obtained by solving the corresponding Bellman equation by modified policy iteration with 100,000 equally spaced grid points (or states).

${ }^{10}$ In the case of Figure 3, the value and policy functions are computed for $x \in[0,300]$ with 300,000 states, and these functions are plotted only for $x \in[0,100]$ to reduce the effect of truncation. 
(a) $\beta=0.1, R=1.7, y=10, \delta=8$ $u(c)=c^{0.3}, 100000$ states
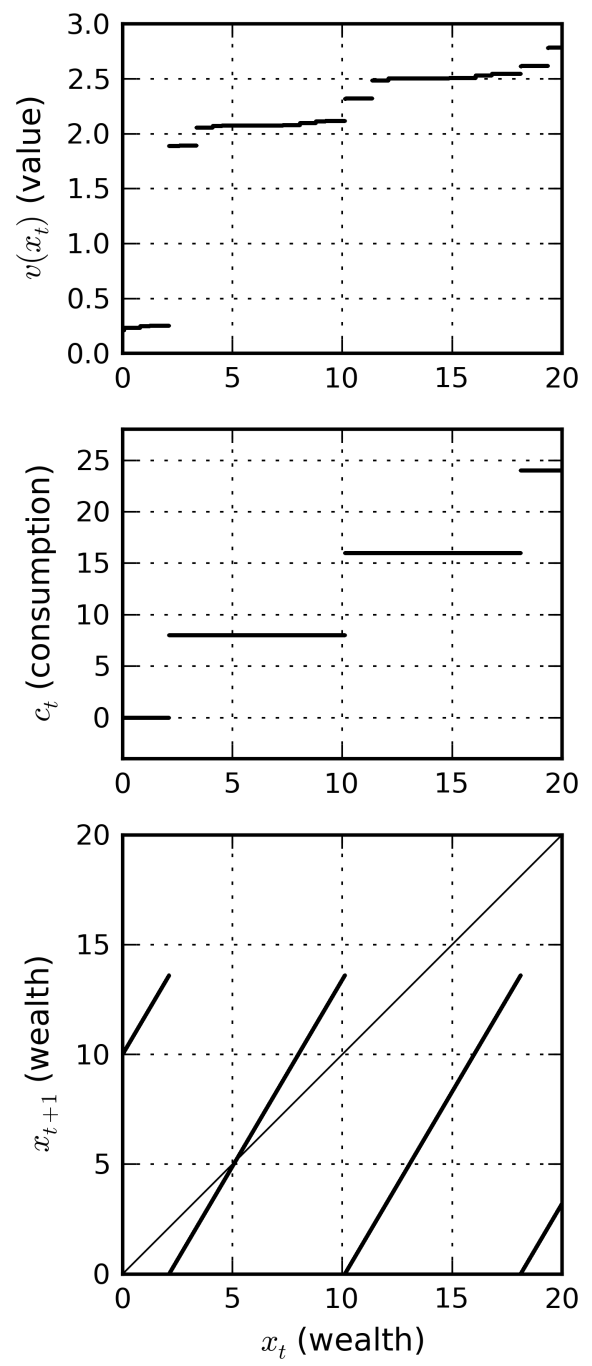

(b) $\beta=0.7, R=1.2, y=10, \delta=5$ $u(c)=c^{0.3}, 100000$ states
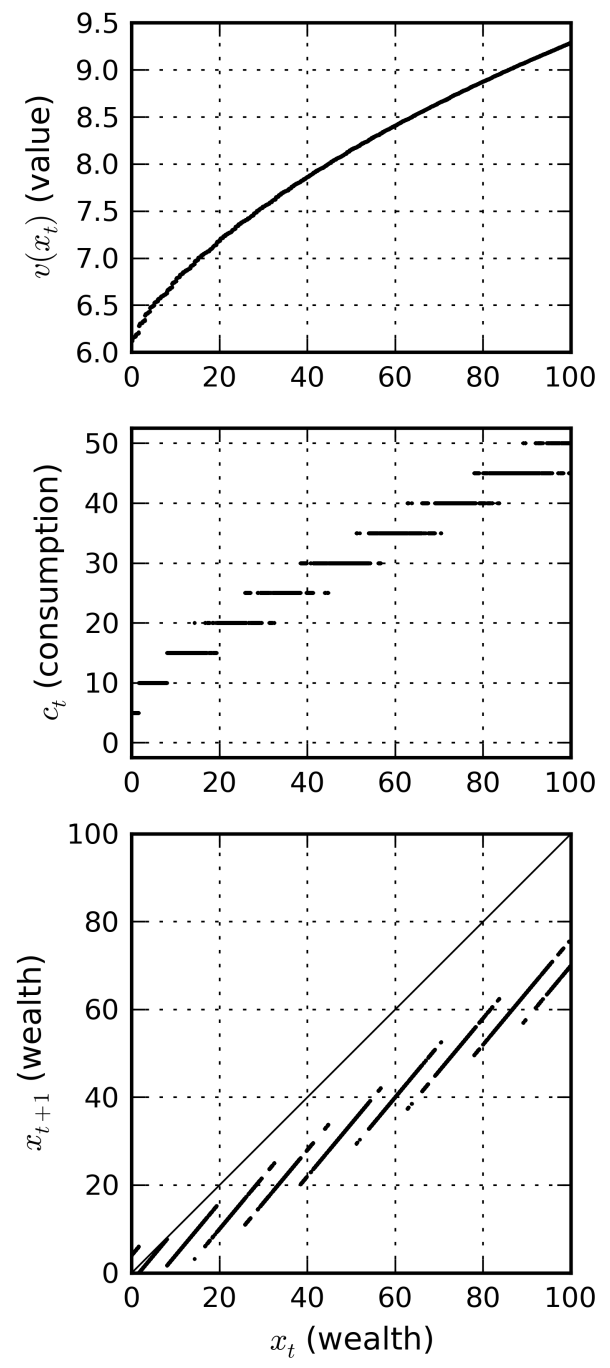

Figure 1: Value functions and policy functions for consumption and wealth 
(a)

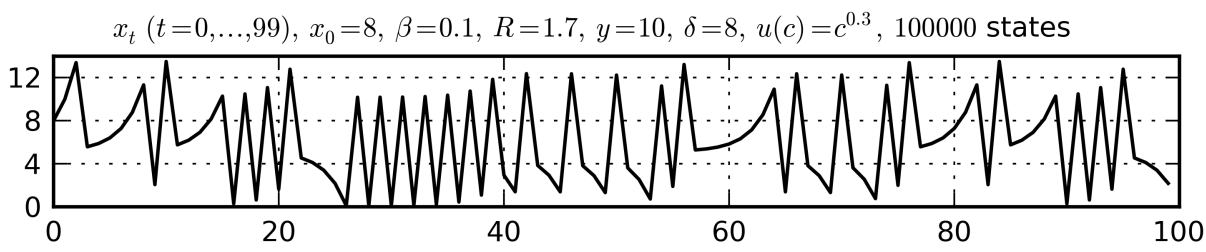

(b)

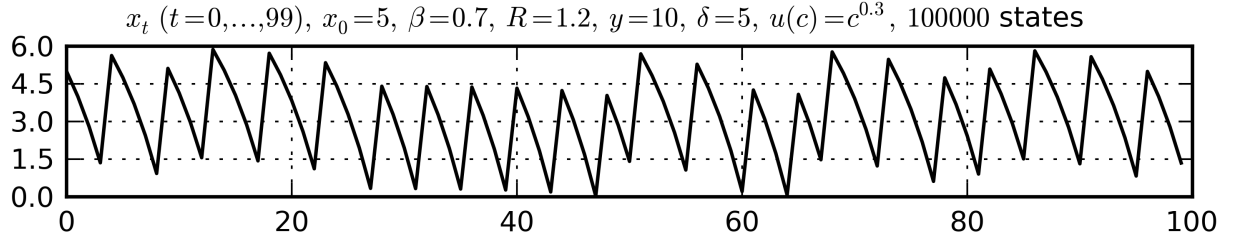

Figure 2: Optimal wealth paths

boundedly and monotonically provided that initial wealth is strictly greater than the nonzero steady state, which is around $15 .^{11}$

It is easy to see that the above analysis can be extended to models with many consumption goods. For example, suppose that $c_{t}$ consists of $m$ goods, i.e., $c_{t}=\left(c_{t}^{1}, c_{t}^{2}, \ldots, c_{t}^{m}\right)$, and that the price of good $i$ is given by $p^{i}$. We can then replace (8) and (11) by

$$
\begin{gathered}
\sum_{i=1}^{m} p^{i} c_{t}^{i}+s_{t}=x_{t}, \\
\forall i \in\{1, \ldots, m\}, \quad c_{t}^{i} \in\left\{0, \delta^{i}, 2 \delta^{i}, 3 \delta^{i}, \ldots\right\},
\end{gathered}
$$

where $\delta^{i}>0$ for each $i$. Constraint (15) means that good $i$ can be purchased only in multiples of $\delta^{i}$. Even in this setting, Theorem 2.1 applies in exactly the same way.

\subsection{Indivisible Durable Goods}

The analysis of the previous subsection can also be extended to models with indivisible durable goods. To be specific, consider the following maximization

\footnotetext{
${ }^{11}$ Since $R>1$, the steady state here is locally unstable, as discussed above.
} 
(a) $\beta=0.9, R=0.5, y=0, \delta=5$ $u(c)=c^{0.3}, 100000$ states
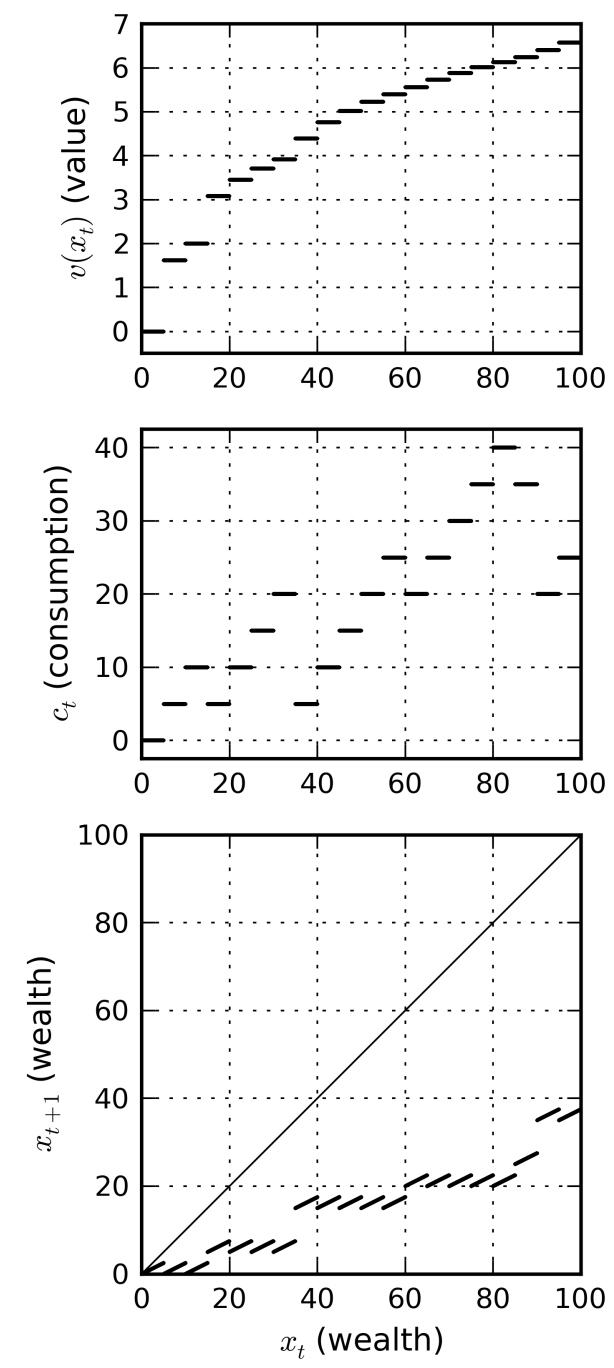

(b) $\beta=0.75, R=1.5, y=0, \delta=5$ $u(c)=c^{0.3}, 100000$ states
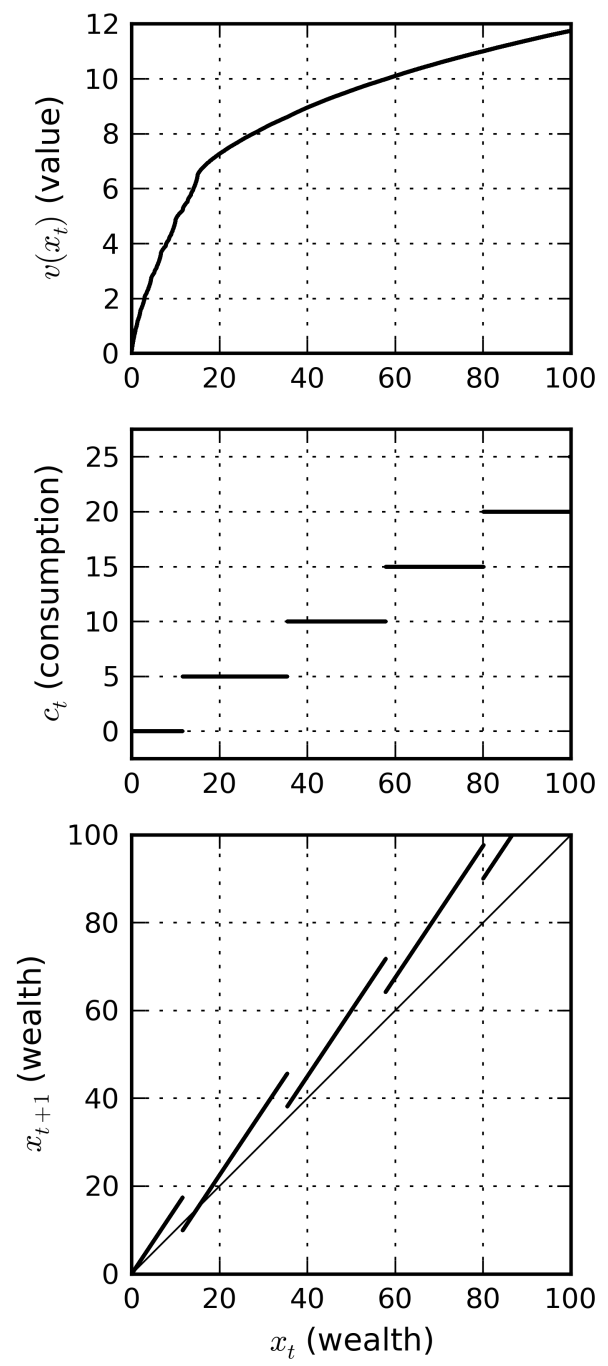

Figure 3: Value functions and policy functions for consumption and wealth 
problem:

$$
\begin{array}{cl}
\max _{\left\{c_{t}, k_{t}, s_{t}, x_{t+1}\right\}_{t=0}^{\infty}} & \sum_{t=0}^{\infty} \beta^{t} u\left(k_{t}\right) \\
\text { s.t. } \forall t \in \mathbb{Z}_{+}, & k_{t}=(1-\gamma) k_{t-1}+c_{t}, \\
& c_{t}+s_{t}=x_{t}, \\
& x_{t+1}=R s_{t}+y, \\
& c_{t} \in\{0, \delta, 2 \delta, 3 \delta, \ldots\} \\
& x_{0}=x \geq 0, k_{-1}=k \geq 0 \text { given, }
\end{array}
$$

where $k_{t}$ is the stock of durable goods at the end of period $t$, and $\gamma \in(0,1]$ is the depreciation rate of durable goods. We assume that the utility function $u: \mathbb{R}_{+} \rightarrow \mathbb{R}$ is strictly increasing. The definitions and assumptions of the other variables and parameters are as in the previous subsection.

Note from (17) that $k_{t}$ can be written as a function of $\left\{c_{t}\right\}$ and $k=k_{-1}$ :

$$
k_{t}=\sum_{i=0}^{t}(1-\gamma)^{i} c_{t-i}+(1-\gamma)^{t+1} k
$$

Hence the objective function can be expressed as a function of $\left\{c_{t}\right\}$ and $k$. Therefore, with $k$ fixed, the maximization problem here takes the form of the right-hand side of (1).

Let $v(x, k)$ be the corresponding value function. Then the argument of the previous subsection shows that $v(x, k)$ is strictly increasing in $x$. Thus by Theorem 2.1 , with $k$ fixed, for almost all $x \geq 0$, any optimal consumption path from $x$ is asymptotically aperiodic.

Although $v(x, k)$ is also strictly increasing in $k$, Theorem 2.1 cannot be used to show the same result in terms of $k$. This is because the theorem requires the objective function to be expressed entirely as a function of $\left\{c_{t}\right\}$. In the current setting the objective function always depends on $k$, so that the problem does not reduce to the right-hand side of (1) (with $x$ fixed). To see why this is important, suppose that $u\left(k_{t}\right)=k_{t}$. In this case, if $\left\{c_{t}\right\}$ is optimal from $k$, then it is also optimal from any $k^{\prime} \geq 0$ since the objective function is additively separable in $\left\{c_{t}\right\}$ and $k$; recall (22). If $\left\{c_{t}\right\}$ happens to be eventually periodic, then this means that there is an eventually periodic optimal consumption path from any $k^{\prime} \geq 0$. 


\section{A Ramsey Model with Linear Utility}

As we mentioned in Subsection 3.2, an asymptotically aperiodic sequence can grow unboundedly and monotonically. In standard neoclassical (or Ramsey) models, however, unbounded growth is ruled out by technology constraints. For example, consider the following maximization problem:

$$
\begin{array}{ll}
\max _{\left\{c_{t}, x_{t+1}\right\}_{t=0}^{\infty}} & \sum_{t=0}^{\infty} \beta^{t} c_{t} \\
\text { s.t. } \forall t \in \mathbb{Z}_{+}, & c_{t}+x_{t+1}=f\left(x_{t}\right), \\
& c_{t} \in H^{\delta} \equiv \begin{cases}\{0, \delta, 2 \delta, 3 \delta, \ldots\} & \text { if } \delta>0, \\
\mathbb{R}_{+} & \text {if } \delta=0, \\
& x_{t+1} \geq 0, \\
& x_{0}=x \geq 0 \text { given, }\end{cases}
\end{array}
$$

where $x_{t}$ is the capital stock at the beginning of period $t$, and $f: \mathbb{R}_{+} \rightarrow \mathbb{R}_{+}$ is the production function, which is assumed to be differentiable on $\mathbb{R}_{++}$, continuous, strictly increasing, strictly concave, and to satisfy

$$
f(0)=0, \quad \lim _{x \downarrow 0} \beta f^{\prime}(x)>1, \quad \lim _{x \uparrow \infty} f^{\prime}(x)<1 .
$$

The last inequality rules out unbounded growth from any initial capital stock. Let $\bar{x}>0$ be the maximum sustainable capital stock, which is given by $f(\bar{x})=\bar{x}$. Note that any feasible capital path from $x \in X \equiv[0, \bar{x}]$ stays in $X$ forever. From here on we restrict ourselves to capital paths in $X$ (though it is not necessary).

Let $x^{*}$ be the unique steady state of the model with $\delta=0$ : i.e., $\beta f^{\prime}\left(x^{*}\right)=$ 1. For $\delta \geq 0$ and $x \in X$, define

$$
F^{\delta}(x)=\left\{y \geq 0: f(x)-y \in H^{\delta}\right\} .
$$

Let $v^{\delta}: \mathbb{R}_{+} \rightarrow \mathbb{R}_{+}$be the value function of the maximization problem (23)$(27)$.

Since $f$ is nonlinear, it is not as easy as in Subsection 3.2 to show that $v^{\delta}$ is strictly increasing. In fact, it is not strictly increasing when $\delta$ is sufficiently large. For example, if $\delta=\bar{x}$, we have $v^{\delta}(x)=0$ for all $x \in[0, \bar{x})$. We can thus expect that $v^{\delta}$ is not strictly increasing when $\delta$ is close to $\bar{x}$. Figure 4(a) 
illustrates such a case. Intuitively, if $\delta$ is close to $\bar{x}$, an optimal capital path spends most of its time near $\bar{x}$, where the slope of the production function is strictly less than one. This means that two optimal capital paths from similar initial capital stocks tend to converge to each other and to have identical consumption paths. As $\delta$ decreases, however, $v^{\delta}$ starts appearing to be a strictly increasing function; see Figure $4(\mathrm{~b}){ }^{12}$

If $\delta$ is small enough, an optimal capital path is expected to stay in a neighborhood of $x^{*}$ in the long run, as in the model with $\delta=0$. Since $f^{\prime}\left(x^{*}\right)=1 / \beta>1$, we can expect that the argument based on the assumption that $R>1$ in Subsection 3.2 can be used to show that $v^{\delta}$ is strictly increasing. This is the idea of the next result. Figure 5 shows that the value function appears to be strictly increasing when $\delta$ is relatively small.

Lemma 4.1. There exists $\bar{\delta}>0$ such that for all $\delta \in(0, \bar{\delta}]$, $v^{\delta}$ is strictly increasing.

Proof. See Appendix B.

The following result is immediate from Lemma 4.1 and Theorem 2.1. ${ }^{13}$

Proposition 4.1. There exists $\bar{\delta}>0$ such that for all $\delta \in(0, \bar{\delta}]$, for almost all $x \in X$, any optimal consumption and capital paths from $x$ are bounded and asymptotically aperiodic.

It is interesting to observe that a decrease in $\delta$ does not necessarily have a significant effect on $v^{\delta}$. Although decreasing $\delta$ from 97 to 60 has a large effect on $v^{\delta}$ (Figure 4), the effect is much less dramatic when $\delta$ is decreased from 60 to 20 (Figures 4(b) and 5(a)), and it appears to be almost negligible when $\delta$ is decreased from 20 to 5 (Figure 5). On the other hand, a change in $\delta$ always has a comparable impact on the optimal policy functions for consumption and capital, as can be seen in Figures 4 and 5 .

Figure 6 illustrates optimal capital paths in the four cases in Figures 4 and 5. While the magnitude of fluctuations is directly related to $\delta$, there appears to be no clear-cut relation between $\delta$ and the frequency of ups and downs.

\footnotetext{
${ }^{12} \mathrm{~A}$ more detailed computation of the value functions in Figures 4(a) and 4(b) suggests that the former is indeed piecewise constant while the latter is strictly increasing.

${ }^{13}$ Recall also footnote 8. Proposition 4.1 does not follow from any argument in Kamihigashi (2000a, 2000b).
} 
(a) $\beta=0.9, \delta=97, f(x)=10 x^{0.5}$ $u(c)=c, 100000$ states
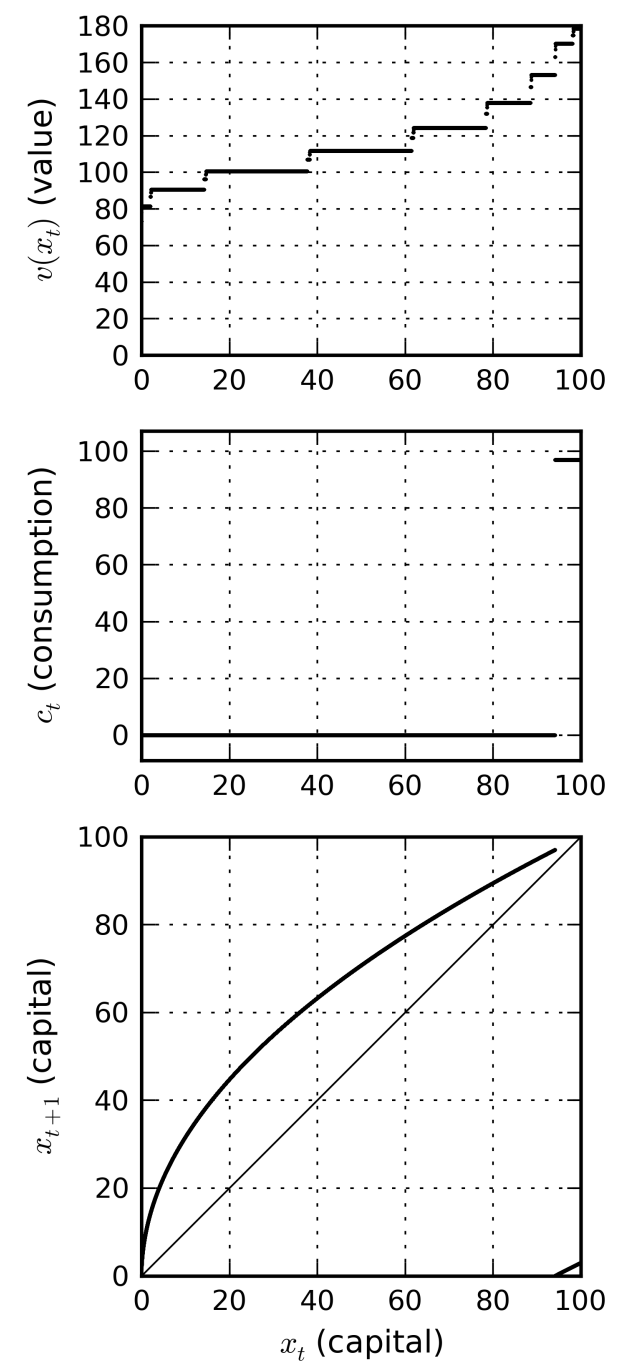

(b) $\beta=0.9, \delta=60, f(x)=10 x^{0.5}$ $u(c)=c, 100000$ states
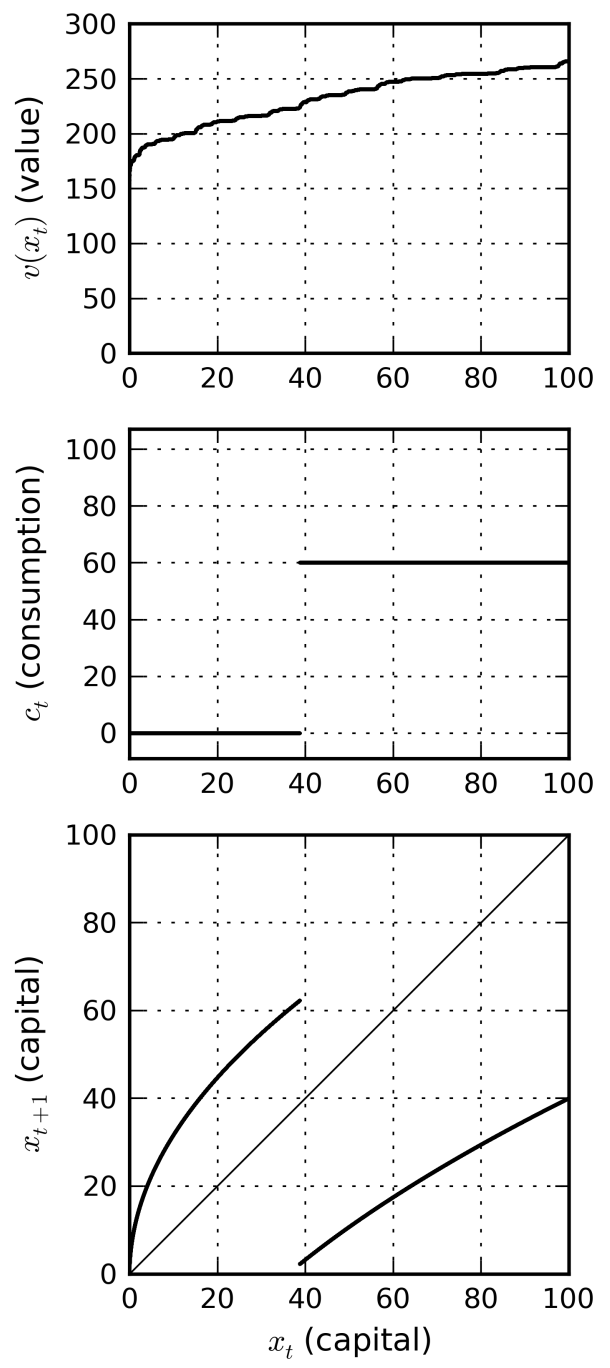

Figure 4: Value functions and policy functions for consumption and capital 
(a) $\beta=0.9, \delta=20, f(x)=10 x^{0.5}$ $u(c)=c, 100000$ states
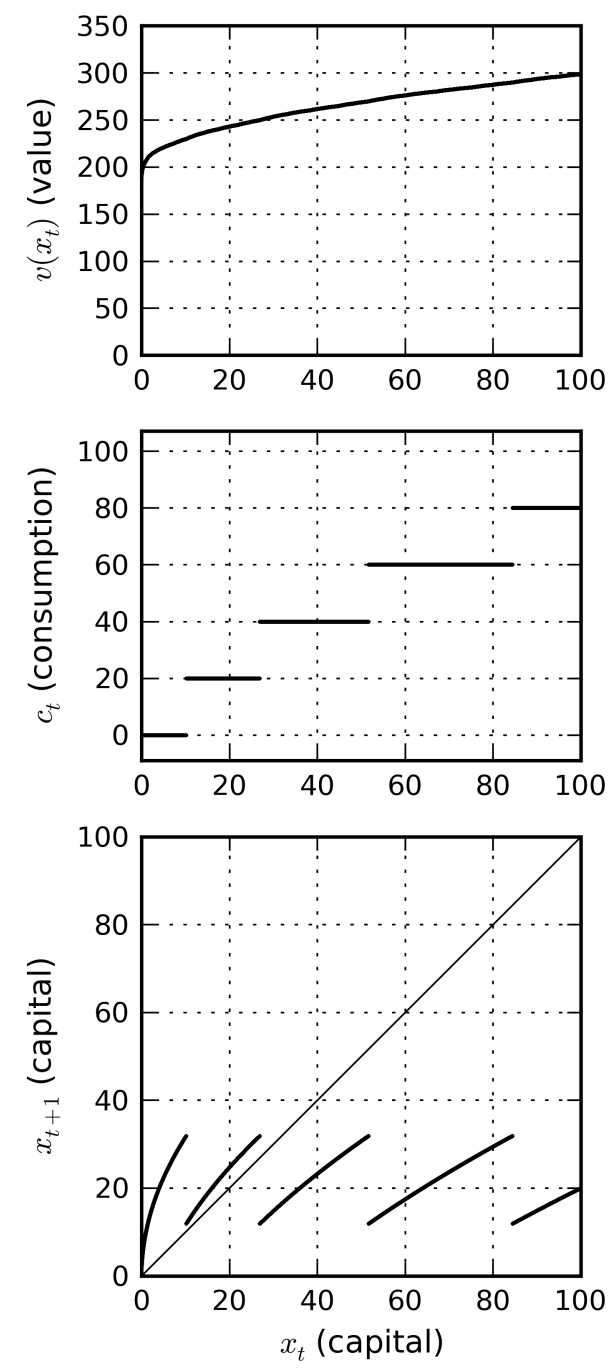

(b) $\beta=0.9, \delta=5, f(x)=10 x^{0.5}$ $u(c)=c, 100000$ states
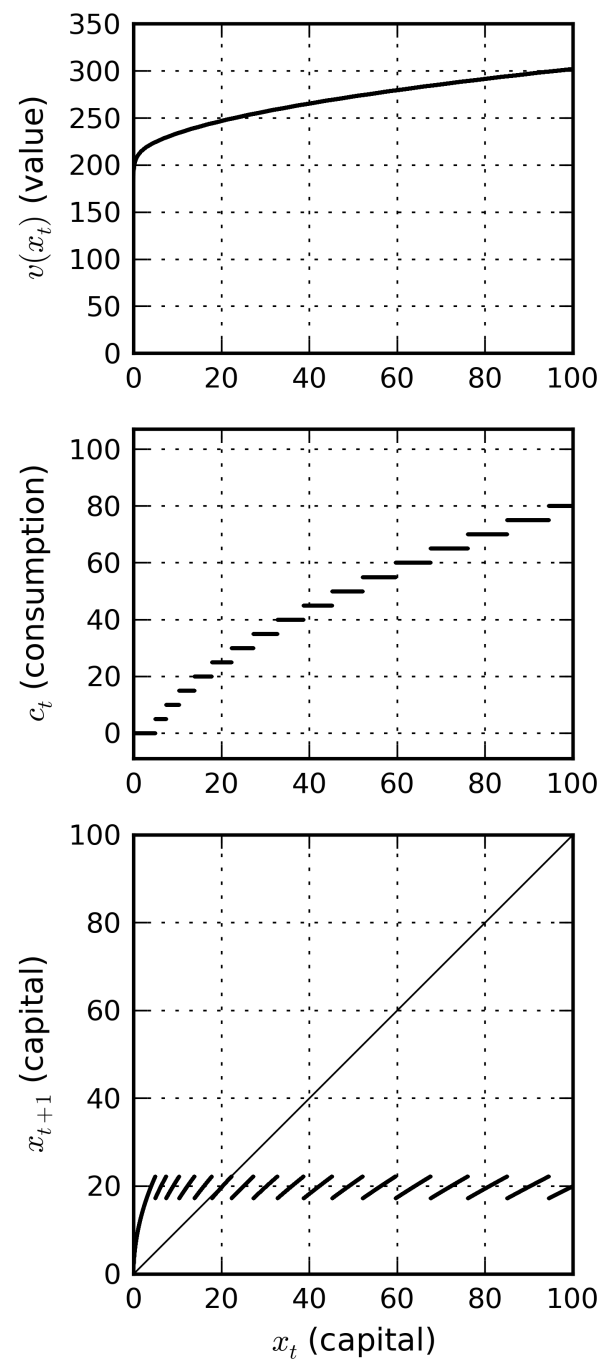

Figure 5: Value functions and policy functions for consumption and capital 
(a)

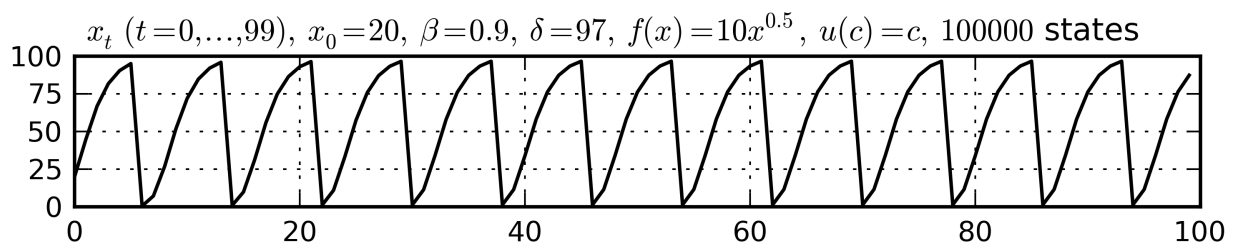

(b)

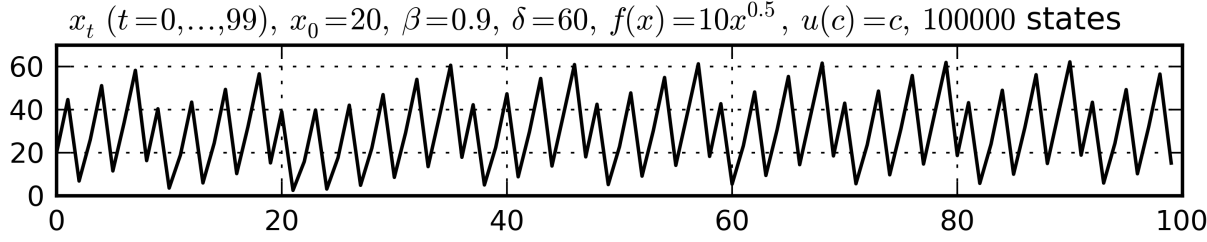

(c)

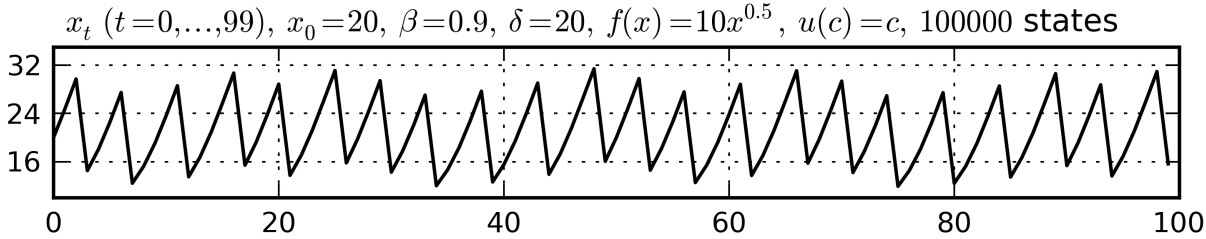

(d)

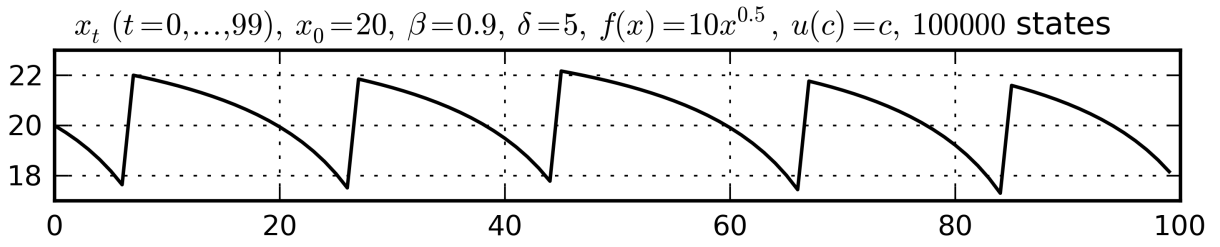

Figure 6: Optimal capital paths

Finally, the idea of Lemma 4.1 seems to work even if the utility function is strictly concave. This point is illustrated with the policy functions for capital in Figure 7, which suggest that even if the utility function is strictly concave, as long as $\delta$ is sufficiently small, any optimal capital path eventually stays close to $x^{*}$, where the slope of the production function is strictly greater than one. If this is the case, $v^{\delta}$ can be shown to be strictly increasing using the argument based on (13). Figure 8 illustrates optimal capital paths in the cases in Figure 7 . These paths roughly remain in the $\delta / 2$-neighborhood of $x^{*}(=20.25)$.

\section{Appendix A Proof of Theorem 2.1}

Lemma A.1. Suppose that $v$ is strictly increasing. Then for any $a \in \mathbb{R}$, we have $\lambda^{n}\left(V_{a}\right)=0$, where $V_{a}=\{x \in X: v(x)=a\}$. 
(a) $\beta=0.9, \delta=10, f(x)=10 x^{0.5}$
$u(c)=c^{0.3}, 100000$ states
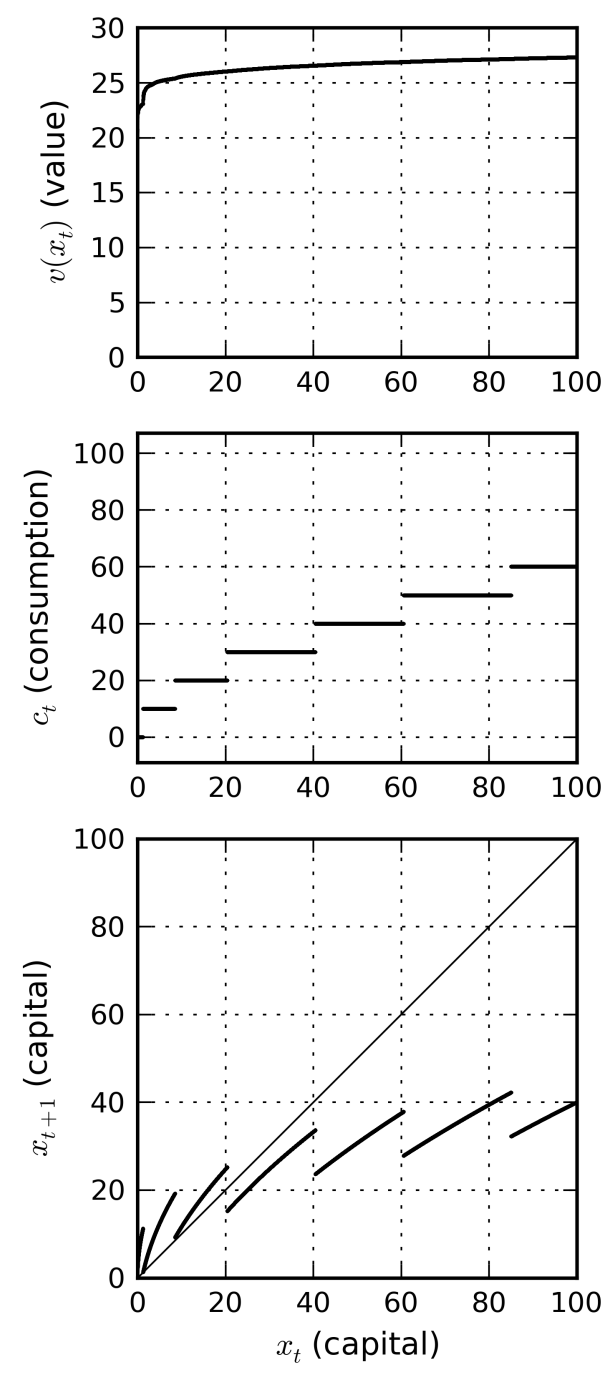

(b) $\beta=0.9, \delta=3, f(x)=10 x^{0.5}$ $u(c)=c^{0.3}, 100000$ states
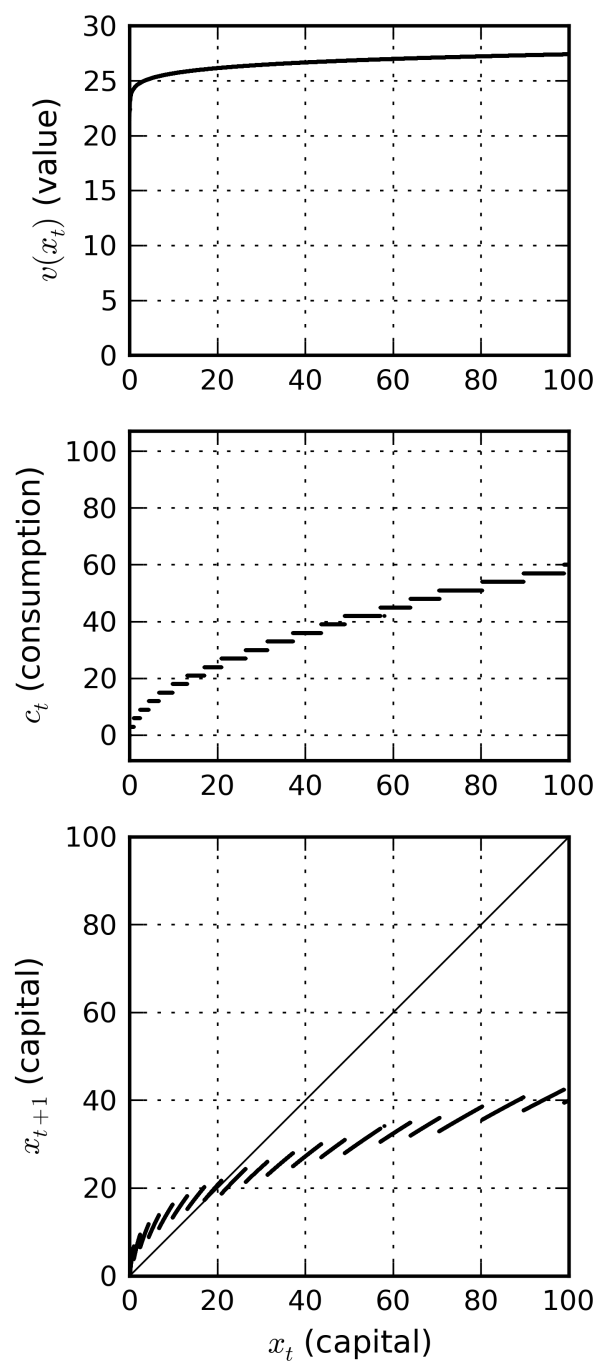

Figure 7: Value functions and policy functions for consumption and capital 
(a)

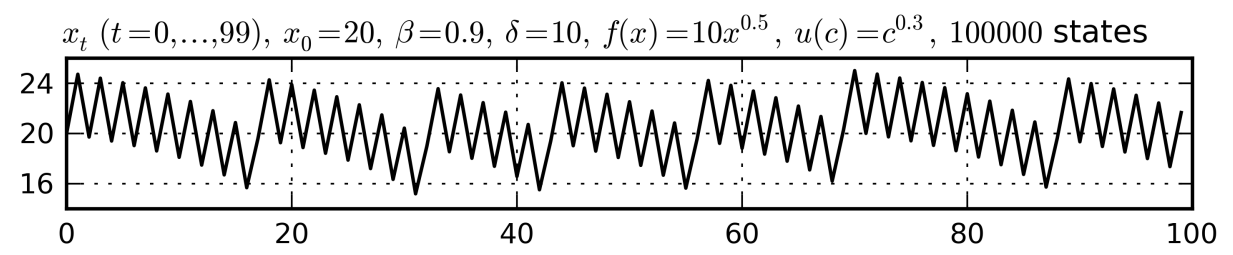

(b)

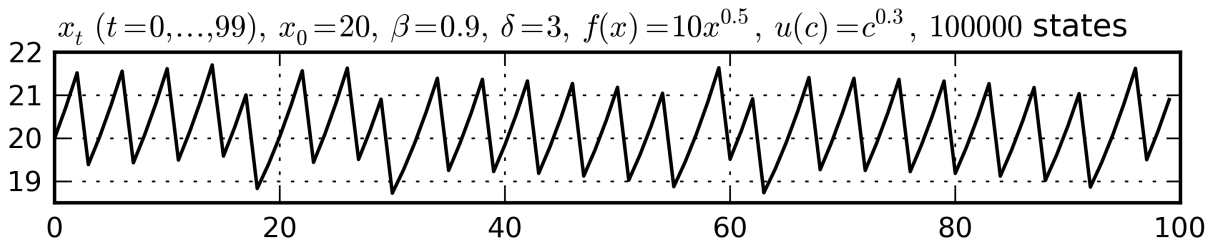

Figure 8: Optimal capital paths

Proof. Let $a \in \mathbb{R}$. Without loss of generality, we extend the domain of $v$ to the entire $\mathbb{R}^{n}$ by defining $v(x)=-\infty$ for $x \in \mathbb{R}^{n} \backslash X$. Since $a \in \mathbb{R}$, this extension does not affect $V_{a}$. We have

$$
\begin{aligned}
\lambda^{n}\left(V_{a}\right) & =\int 1\{v(x)=a\} d \lambda^{n}(x) \\
& =\int \cdots \int 1\left\{v\left(x_{1}, x_{2}, \ldots, x_{n}\right)=a\right\} d \lambda\left(x_{1}\right) \cdots d \lambda\left(x_{n}\right),
\end{aligned}
$$

where $1\{\cdot\}$ is the indicator function, ${ }^{14}$ and (31) holds by the Tonelli-Fubini theorem (Dudley, 2002, p. 139). Since $v$ is strictly increasing, given $\left(x_{2}, \ldots, x_{n}\right) \in$ $\mathbb{R}^{n-1}$, we have $1\left\{v\left(x_{1}, x_{2}, \ldots, x_{n}\right)=a\right\}=0$ for almost all $x_{1} \in \mathbb{R}$; thus

$$
\int 1\left\{v\left(x_{1}, x_{2}, \ldots, x_{n}\right)=a\right\} d \lambda\left(x_{1}\right)=0 .
$$

Substituting this into (31) yields the lemma.

Lemma A.2. $\Pi \equiv\left\{\left\{c_{t}\right\} \in K^{\infty}:\left\{c_{t}\right\}\right.$ is eventually periodic $\}$ is countable. ${ }^{15}$

Proof. Let $\Gamma=\cup_{i, j \in \mathbb{N}} K^{i} \times K^{j}$. Define $\Psi: \Pi \rightarrow \Gamma$ as follows: for $c \equiv\left\{c_{t}\right\} \in \Pi$, let $i_{c}$ be the smallest $i \in \mathbb{N}$ such that $\left\{c_{t}\right\}_{t=i}^{\infty}$ is periodic; let $j_{c}$ be the smallest $j \in \mathbb{N}$ such that $\forall t \geq i_{c}, c_{t}=c_{t+j}$; and define

$$
\Psi\left(\left\{c_{t}\right\}\right)=\left(\left\{c_{t}\right\}_{t=0}^{i_{c}-1},\left\{c_{t}\right\}_{t=i_{c}}^{i_{c}+j_{c}-1}\right) \in K^{i_{c}} \times K^{j_{c}} \subset \Gamma
$$

\footnotetext{
${ }^{14}$ That is, $1\{v(x)=a\}=1$ if $v(x)=a$, and $1\{v(x)=a\}=0$ otherwise

${ }^{15}$ This result is adapted from Kamihigashi (2000a, Lemma 6). A similar argument is used in Kamihigashi (2000b, Lemma 5.14).
} 
Clearly $\Psi$ is one-to-one. Since the countable union of countable sets is countable, $\Gamma$ is countable. Therefore $\Pi$ is also countable.

To prove Theorem 2.1, suppose that $v$ is strictly increasing. Define

$$
A=\{a \in \mathbb{R}: \exists c \in \Pi \cap D, w(c)=a\} .
$$

Since $\Pi$ is countable by Lemma A.2, $A$ is also countable. Define

$$
Z=\cup_{a \in A} V_{a}=\{x \in X: v(x) \in A\} .
$$

Since $A$ is countable and the $V_{a}$ are disjoint,

$$
\lambda^{n}(Z)=\sum_{a \in A} \lambda^{n}\left(V_{a}\right)=0
$$

where the second equality holds by Lemma A.1. Let $x \in X \backslash Z$ and $c \in$ $C^{*}(x)$. We have $w(c)=v(x) \notin A$, which implies $c \notin \Pi$ by (34). Thus $c$ is asymptotically aperiodic. This establishes that $Z$ has the desired properties.

To prove Corollary 2.1, replace the first equality in (36) with $\leq$. Note that the second equality in (36) holds by (3). The rest of the proof remains the same.

\section{Appendix B Proof of Lemma 4.1}

Note that for $\delta \geq 0$ and $x \in X$, we have the Bellman equation

$$
\begin{aligned}
v^{\delta}(x) & =\max _{y \in F^{\delta}(x)}\left\{f(x)-y+\beta v^{\delta}(y)\right\} \\
& =f(x)+\max _{y \in F^{\delta}(x)}\left\{\beta v^{\delta}(y)-y\right\} .
\end{aligned}
$$

Let $G^{\delta}$ be the optimal policy correspondence:

$$
G^{\delta}(x)=\underset{y \in F^{\delta}(x)}{\operatorname{argmax}}\left\{f(x)-y+\beta v^{\delta}(y)\right\}=\underset{y \in F^{\delta}(x)}{\operatorname{argmax}}\left\{\beta v^{\delta}(y)-y\right\} .
$$

Lemma B.1. $v^{0}: \mathbb{R}_{+} \rightarrow \mathbb{R}_{+}$is continuous and strictly concave. Furthermore, $x^{*}$ is the unique solution to $\max _{y \geq 0}\left[\beta v^{0}(y)-y\right]$ :

$$
\forall y \in \mathbb{R}_{+} \backslash\left\{x^{*}\right\}, \quad \beta v^{0}(y)-y<\beta v^{0}\left(x^{*}\right)-x^{*} .
$$


Proof. Standard arguments show that $v^{0}$ is continuous and strictly concave. Let $x_{0} \geq x^{*}$. Then the capital path $\left\{x_{t+1}\right\}$ given by $x_{t}=x^{*}$ for all $t \in \mathbb{N}$ is feasible and satisfies the Euler equation and the transversality condition:

$$
1=\beta f^{\prime}\left(x_{t+1}\right), \quad \lim _{t \uparrow \infty} \beta^{t} f^{\prime}\left(x_{t+1}\right) x_{t+1}=0 .
$$

By strict concavity, $\left\{x_{t+1}\right\}$ is the unique optimal capital path from $x_{0}$; thus $G^{0}\left(x_{0}\right)=\left\{x^{*}\right\}$, i.e., $x^{*}=\operatorname{argmax}_{y \in\left[0, f\left(x_{0}\right)\right]}\left\{\beta v^{0}(y)-y\right\}$. Since $v^{0}$ is strictly concave, $\beta v^{0}(y)-y$ is strictly decreasing in $y>f\left(x_{0}\right) \geq x^{*}$. Thus (40) holds.

Since the definitions of feasibility and optimality depend on $\delta$, we make explicit the dependence on $\delta$ by saying that a path is $\delta$-feasible, etc.

Lemma B.2. For any $\delta>0$, we have

$$
\forall x \in X, \quad v^{\delta}(x) \geq v^{0}(x)-\delta /(1-\beta) .
$$

Proof. Let $\delta>0$ and $x \in X$. Let $\left\{c_{t}\right\}$ be a 0-optimal consumption path from $x$. For $t \in \mathbb{Z}_{+}$, define $c_{t}^{\delta}=\max \left\{c \in H^{\delta}: c \leq c_{t}\right\}$. Then $\left\{c_{t}^{\delta}\right\}$ is $\delta$-feasible from $x$, and $c_{t}^{\delta} \geq c_{t}-\delta$ for all $t \in \mathbb{Z}_{+}$. We have

$$
v^{\delta}(x) \geq \sum_{t=o}^{\infty} \beta^{t} c_{t}^{\delta} \geq \sum_{t=0}^{\infty} \beta^{t}\left(c_{t}-\delta\right)=v^{0}(x)-\delta /(1-\beta) .
$$

Now (42) follows.

Lemma B.3. For any $\hat{x}>x^{*}$, there exists $\bar{\delta}>0$ such that

$$
\forall \delta \in(0, \bar{\delta}], \forall x \in X, \forall y \in G^{\delta}(x), \quad y+\delta<\hat{x} .
$$

Proof. Suppose that the lemma is false. Then there exist $\hat{x}>x^{*},\left\{\delta_{i}\right\}_{i=1}^{\infty} \subset$ $\left(0, \hat{x}-x^{*}\right)$ with $\delta_{i} \downarrow 0,\left\{x_{i}\right\}_{i=1}^{\infty} \subset X$, and $\left\{y_{i}\right\}_{i=1}^{\infty} \subset X$ such that

$$
\forall i \in \mathbb{N}, \quad y_{i} \in G^{\delta_{i}}\left(x_{i}\right), \quad y_{i}+\delta_{i} \geq \hat{x} .
$$

Taking a subsequence, we may assume that $y_{i}$ converges to some $y^{*} \in[\hat{x}, \bar{x}]$.

Let $i \in \mathbb{N}$. Since $v^{0} \geq v^{\delta_{i}}$, we have

$$
\beta v^{0}\left(y_{i}\right)-y_{i} \geq \beta v^{\delta_{i}}\left(y_{i}\right)-y_{i}
$$


Note from (45) that $y_{i} \geq \hat{x}-\delta_{i}>x^{*}$. Thus $\left\{y \in F^{\delta_{i}}\left(x_{i}\right): y \geq x^{*}\right\} \neq \emptyset$. Define $\tilde{y}_{i}=\min \left\{y \in F^{\delta_{i}}\left(x_{i}\right): y \geq x^{*}\right\} \in\left[x^{*}, x^{*}+\delta_{i}\right]$; note that $\tilde{y}_{i} \leq y_{i}$. We have

$$
\beta v^{\delta_{i}}\left(y_{i}\right)-y_{i} \geq \beta v^{\delta_{i}}\left(\tilde{y}_{i}\right)-\tilde{y}_{i} \geq \beta\left[v^{0}\left(\tilde{y}_{i}\right)-\delta_{i} /(1-\beta)\right]-\tilde{y}_{i},
$$

where the second inequality uses Lemma B.2. It follows from (46) and (47) that

$$
\beta v^{0}\left(y_{i}\right)-y_{i} \geq \beta\left[v^{0}\left(\tilde{y}_{i}\right)-\delta_{i} /(1-\beta)\right]-\tilde{y}_{i} .
$$

Since $y_{i} \rightarrow y^{*} \geq \hat{x}$ and $\tilde{y}_{i} \rightarrow x^{*}$, letting $i \uparrow \infty$ in (48) and recalling the continuity of $v^{0}$, we obtain

$$
\beta v^{0}\left(y^{*}\right)-y^{*} \geq \beta v^{0}\left(x^{*}\right)-x^{*}
$$

which contradicts Lemma B.1. This completes the proof.

For the rest of the proof, let $\hat{x}>x^{*}$ be such that $f^{\prime}(\hat{x})>1$, and let $\bar{\delta}>0$ satisfy (44).

Lemma B.4. Let $\delta \in(0, \bar{\delta}], x \in X$, and $x^{\prime}>x$. Let $\left\{c_{t}\right\}$ be a $\delta$-optimal consumption path from $x$. Then there exists a $\delta$-feasible consumption path $\left\{c_{t}^{\prime}\right\}$ from $x^{\prime}$ such that

$$
\text { (i) } \forall t \in \mathbb{Z}_{+}, c_{t}^{\prime} \geq c_{t}, \quad \text { (ii) } \exists t \in \mathbb{Z}_{+}, c_{t}^{\prime}>c_{t} .
$$

Proof. Define $\left\{x_{t}^{\prime}\right\}$ recursively by $x_{t+1}^{\prime}=f\left(x_{t}^{\prime}\right)-c_{t}$. Suppose that there exists no $\left\{c_{t}^{\prime}\right\}$ satisfying (50). Then we must have

$$
\forall t \in \mathbb{Z}_{+}, \quad x_{t+1}^{\prime}<x_{t+1}+\delta<\hat{x} .
$$

The second inequality above holds by (44). The first inequality holds since if $x_{s+1}^{\prime} \geq x_{s+1}+\delta$ for some $s \in \mathbb{Z}_{+}$, then $c_{s}$ can be increased by $\delta$ without decreasing $c_{t}$ with $t \neq s$. For $t \in \mathbb{N}$, by concavity of $f$ and (51),

$$
x_{t+1}^{\prime}-x_{t+1}=f\left(x_{t}^{\prime}\right)-f\left(x_{t}\right) \geq f^{\prime}\left(x_{t}^{\prime}\right)\left(x_{t}^{\prime}-x_{t}\right) \geq f^{\prime}(\hat{x})\left(x_{t}^{\prime}-x_{t}\right) .
$$

Since $f^{\prime}(\hat{x})>1$, it follows that $x_{t}^{\prime}-x_{t} \rightarrow \infty$, contradicting (51).

Lemma 4.1 now follows from Lemma B.4. 


\section{References}

Aguirregabiria, Victor and Pedro Mira (2010) Dynamic discrete choice structural models: a survey. Journal of Econometrics 156, 38-67.

Aoki, Masanao (1998) Simple model of asymmetrical business cycles: interactive dynamics of a large number of agents with discrete choices. Macroeconomic Dynamics 2, 427-442.

Bischi, Gian-Italo, Mauro Gallegati, Laura Gardini, Roberto Leombruni, and Antonio Palestrini (2006) Herd behavior and nonfundamental asset price fluctuations in financial markets. Macroeconomic Dynamics 10, 502-528.

Chabrillac, Yves and J.-P. Crouzeix (1987) Continuity and differentiability properties of monotone real functions of several variables. Mathematical Programming Study 30, 1-16.

Dechert, Davis W. and Kazuo Nishimura (1983) A complete characterization of optimal growth paths in an aggregate model with a non-concave production function. Journal of Economic Theory 31, 332-354.

Dudley, R.M. (2002) Real Analysis and Probability. Cambridge: Cambridge University Press.

Kamihigashi, Takashi (2000a) The policy function of a discrete-choice problem is a random number generator. Japanese Economic Review 51, 5171 .

Kamihigashi, Takashi (2000b) Indivisible labor implies chaos. Economic Theory $15,585-598$.

Kamihigashi, Takashi and Santanu Roy (2006) Dynamic optimization with a nonsmooth, nonconvex technology: the case of a linear objective function, Economic Theory 29, 325-340.

Kamihigashi, Takashi and Santanu Roy (2007) A nonsmooth, nonconvex model of optimal growth. Journal of Economic Theory 132, 435-460.

Keane, Michael P. and Kenneth I. Wolpin (2009) Empirical applications of discrete choice dynamic programming models. Review of Economic Dynamics 12, 1-22. 
Nishimura, Kazuo and Makoto Yano (1995) Non-linear dynamics and chaos in optimal growth: an example. Econometrica 63, 981-1001.

Nishimura, Kazuo, Gerhard Sorger, and Makoto Yano (1994) Ergodic chaos in optimal growth models with low discount rates. Economic Theory 4, 705-717.

Vergrugge, Randal (2003) Interactive-agent economies: an elucidative framework and survey of results. Macroeconomic Dynamics 7, 424-472. 